\title{
Aplicación plural de herramientas para gamificar. Análisis y comparativa
}

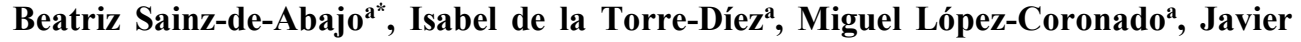 \\ M. Aguiar Pérez ${ }^{a}$ y Carlos de Castro Lozano ${ }^{b}$ \\ ${ }^{a}$ Departamento de Teoría de la Señal, Comunicaciones e Ingeniería Telemática. Universidad de \\ Valladolid. Paseo de Belén, 15, 47011 - Valladolid. E-mail: beasai@tel.uva.es”, isator@tel.uva.es, \\ miglop@tel.uva.es, javagu@tel.uva.es
}

bepartamento de Informática y Análisis Numérico. Universidad de Córdoba. Carretera MadridCádiz Km.396-A, 14071-Córdoba. E-mail: ma1caloc@uco.es

\begin{abstract}
The benefits of gamification, as a pedagogical instrument for the motivation of students in their learning process, are demonstrated easily. This playful approach, based on the competitiveness offered by the game, pursues the student's interest in the subject, placing him in a more active role and stimulating him through rewards. There are many tools that offer us to gamify. The aim of this paper is the comparative analysis of some of the most well-known applications for the realization of online tests like KAHOOT!, SOCRATIVE, QUIZIZZ or GOOGLEFORMS. This allows us to consider the most appropriate in each case for the contents evaluated in the classroom.
\end{abstract}

Keywords: gamification, KAHOOT!, SOCRATIVE, QUIZIZZ, GOOGLEFORMS, competition, training, self-examination, motivation, reward.

\begin{abstract}
Resumen
Los beneficios de la gamificación, como instrumento pedagógico para la motivación de los alumnos en su proceso de aprendizaje, están sobradamente demostrados. Este enfoque lúdico, basado en la competitividad que ofrece el juego, persigue el interés del discente por la materia, colocándole en un rol más activo y estimulándole a través de recompensas. Son muchas las herramientas que nos ofrecen para gamificar. El objetivo de este articulo es el análisis comparativo de algunas de las aplicaciones más conocidas para la realización de test en línea como KAHOOT!, SOCRATIVE, QUIZIZZ o GOOGLEFORMS. Ello nos permite considerar la más adecuada en cada caso para los contenidos evaluados en el aula.
\end{abstract}

Palabras clave: gamificación, KAHOOT!, SOCRATIVE, QUIZIZZ, GOOGLEFORMS, competición, entrenamiento, autoevaluación, motivación, recompensa. 


\section{Introducción}

El uso de smartphones, tabletas u ordenadores portátiles en la educación actual ha derivado en el desarrollo de una rama del conocimiento, acuñada como m-learning (IzquierdoAlvarez et al., 2018). Con el propósito de aprender es cada vez más común la distribución entre los escolares de dispositivos móviles, para usar en cualquier momento y lugar (Song, 2014). Y se observa de forma palpable el esfuerzo de las empresas e instituciones en el desarrollo de aplicaciones que apoyen el Bring Your Own Device (BYOD). Los millennials y la generación $\mathrm{Z}$, todos ellos nativos digitales, aplican las TIC en todas las facetas de su vida. También los docentes estamos cada vez más concienciados en su necesidad de uso. Por ello lo más natural es su aplicación, como soporte y método de instrucción, para maximizar el aprendizaje dentro y fuera del aula. Cuando observamos a los alumnos prescindir del boli y papel, teniendo como única herramienta para el seguimiento de la clase un portátil o tableta, pedirles que prescindan de sus dispositivos electrónicos resulta anacrónico.

La reforma e innovación en la educación superior conllevan un cambio en la evaluación del aprendizaje de los estudiantes (Guardia et al., 2019). Lo lúdico potencia la motivación, la concentración y el esfuerzo. Un ejemplo de éxito de esta tendencia, introducir el juego en un entorno educativo, lo vemos en la web https://www.duolingo.com/ La gamificación puede tener un impacto importante en estudiantes y profesores al integrar los elementos del juego en la jornada laboral, mejorando así el aprendizaje y la enseñanza (Fuß et al., 2014).

Es necesario habilitar mecanismos para que, a pesar del absentismo que a priori se espera en el aula los días planificados como clase magistral, se alcancen los objetivos previstos adaptando la metodología (Sainz et al., 2018). Si incentivamos al alumno y reconocemos, aunque sea mínimamente, su asistencia, puntualidad y atención en el aula, estaremos seguros de garantizar su presencia.

\section{Objetivo}

Los autores proponemos implementar un proceso de evaluación de aprendizaje participativo y autoevaluación a través de diferentes herramientas, conociendo sus particularidades y los beneficios que nos ofrecen. Para el análisis y comparación se seleccionaron las aplicaciones gestoras gratuitas KAHOOT! (https://kahoot.com/), SOCRATIVE (https://www.socrative.com/), QUIZIZZ (https://quizizz.com/) y GOOGLEFORMS (https://www.google.com/forms/about/). El porqué de la elección de éstas frente a otras, está directamente relacionado con los trabajos que avalan de forma positiva la influencia motivadora de estos cuestionarios en línea en el sector educativo (Barnes, 2017; Chaiyo \& Nokham, 2017; Garcia-Garcia et al., 2017; Llerena y Rodriguez, 2017; Wang y Lieberoth, 2016; Wee y Choong, 2019).

Si además se les premia con incentivos como la mejora de la calificación en la evaluación final, puntuando más que los compañeros en el juego, además de entretenido se dispara el ánimo de competición, y esa actitud en sí predispone para el aprendizaje (Iwamoto et al., 
2017; Jurgelaitis et al., 2019). También la sana competencia entre los estudiantes puede resultar un acicate para dedicar tiempo al estudio (Sainz et al., 2018).

\section{Desarrollo de la innovación}

\subsection{Contexto}

"Recursos Documentales e Informáticos" se imparte en la Universidad de Valladolid. Forma parte del Curso de Adaptación al Grado en Criminología. Se trata de una asignatura de carácter básico, de 6 créditos ECTS y que, además de que el alumno domine conceptos relacionados con la misma, persigue competencias transversales como son la capacidad de crítica, síntesis y análisis de los datos.

Los alumnos que los últimos años han solicitado el ingreso pertenecen, en su totalidad, a los cuerpos y fuerzas de seguridad del Estado. La mayoría disponen de un Título Propio en Criminología y esperan obtener el título habilitante de Grado, para poder ascender en la escala profesional a plazas a las que están vetados, por no contar con una acreditación oficial reconocida por el Estado Español.

Son alumnos que a pesar de estar muy motivados, en algunos casos vuelven al aula después de muchos años y presentan dificultades de comprensión ante las nuevas herramientas y conceptos impartidos. Varios estudiantes del curso 2018-2019 contaban con más de cincuenta años. La dificultad como docentes es que la transmisión de la materia recogida en el programa llegue de forma clara y que la interioricen. Es nuestra misión buscar la mejor estrategia para vehicular los contenidos y que necesariamente tienen que conocer y asimilar. Y para ello, y en beneficio de los alumnos, desde el Espacio Europeo de Educación Superior (EEES) se nos sugiere técnicas pedagógicas que logren ese fin. Tambien las universidades invierten en ayudas, a través de Programas de Innovación Docente, que facilitan la adaptación de la metodología tradicional con nuevas técnicas para la mejora de los resultados del alumnado.

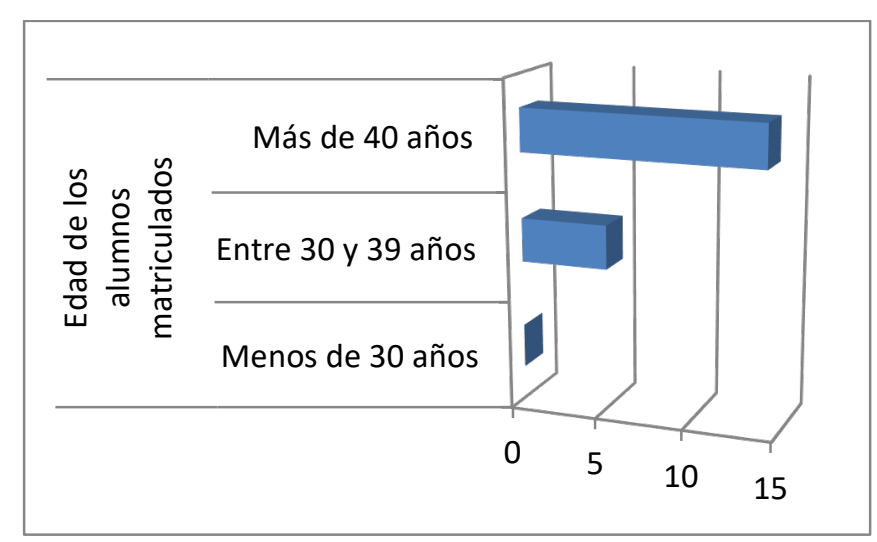

Fig. 1 Edad de los 19 alumnos que participaron en la experiencia 
Teniendo en cuenta que los alumnos ejercen su profesión a tiempo completo y estando los contenidos accesibles en la plataforma del Campus Virtual de la institución, el absentismo resulta tentador. Por ello, para que sirva de acicate, conviene programar actividades con un sistema de recompensas. Como resultado se constata que el alumno acude al aula, aunque sea para tener la opción de arañar algún punto que le beneficie en la evaluación final, y porque la clase le resulta más llevadera.

\subsection{Procedimiento}

La metodología contó con una serie de fases que se resumen en la Figura 2:

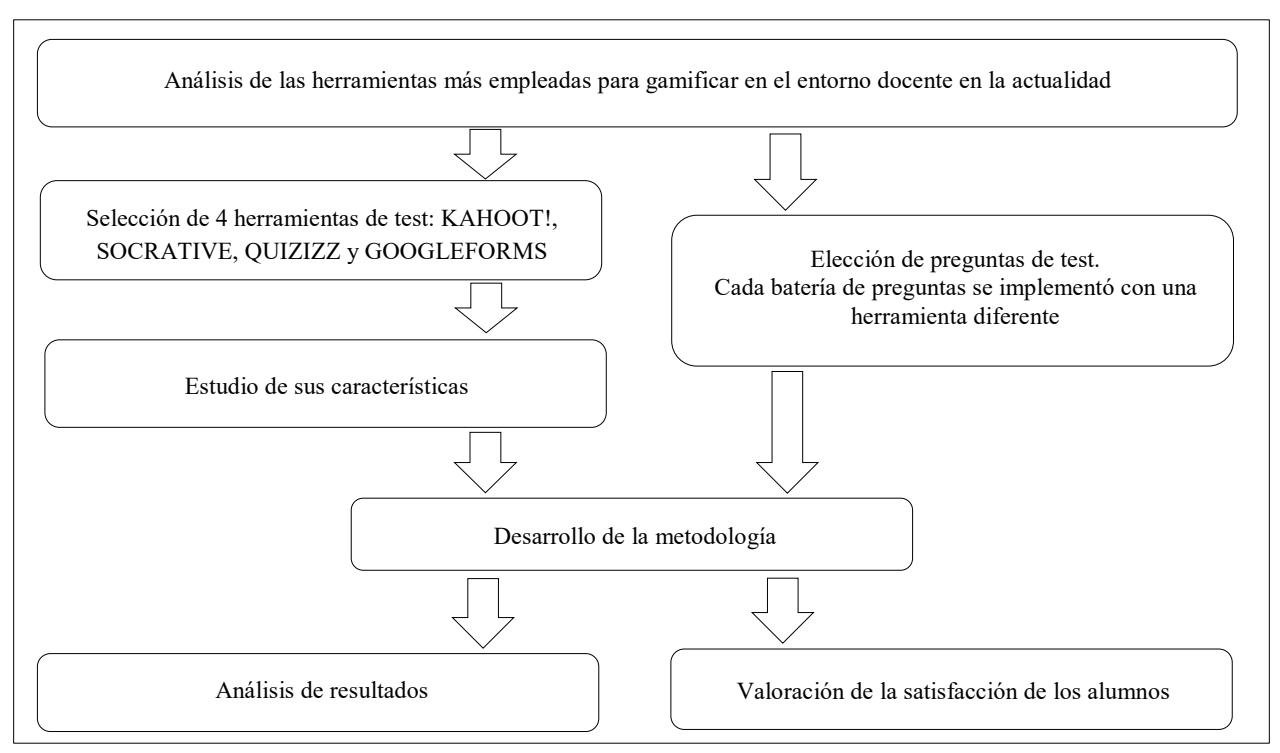

Fig. 2 Diagrama de flujo de la metodología implementada

Como punto de partida, y teniendo en cuenta la experiencia capitalizada de cursos anteriores tras gamificar con Kahoot!, se hizo una búsqueda de las herramientas de acceso libre para el desarrollo de encuestas en línea. Las lecturas y conclusiones se pusieron en común en el grupo de trabajo de los docentes implicados para decidir, previo al inicio del curso, las que finalmente se implementarían y analizar y comparar sus características más destacadas. A continuación de decidió la batería de preguntas de cada tema, con diverso grado de dificultad, y con cada una se generó la encuesta mediante las diferentes herramientas.

Algunos test se realizaron en el aula como evaluación participativa. Otros se diseñaron para una autoevaluación individual, facilitando un enlace y estableciendo una fecha límite, sirviendo de repaso y fijando los conceptos más importantes. El grado de acierto a las preguntas nos facilita conocer si los contenidos son entendibles.

Como criterio de elección de las herramientas seleccionadas para el desarrollo de los test en línea se escogieron, tras el análisis de la numerosa bibliografía consultada, aquellas que son 
sencillas de manejar y permiten participar sin necesidad, a priori, de conocer la herramienta al ser intuitivas. Además de ser gratuitas están en continua evolución incorporando funcionalidades nuevas.

\subsection{Análisis del grado de satisfacción}

Finalmente y gracias a la opinión de los estudiantes, recogida mediante una encuesta voluntaria y anónima, que fue completada por la totalidad de los alumnos matriculados, se pudo valorar la experiencia.

La encuesta (ver Fig. 3) cuenta con una serie de preguntas que se puntúan en una escala de 5 niveles de percepción (siendo 1-Muy malo y 5-Muy bueno) y 3 preguntas abiertas.

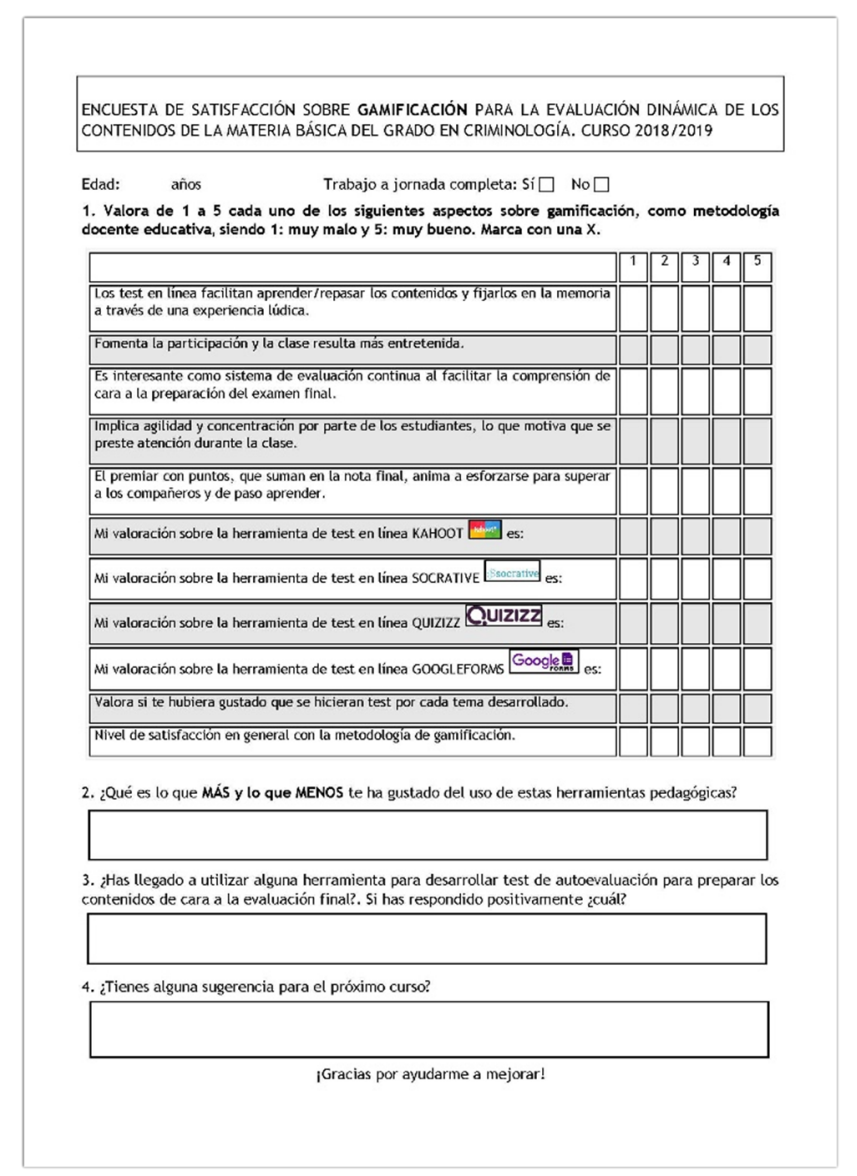

Fig. 3 Encuesta de satisfacción sobre diferentes herramientas de gamificación

\section{Resultados}

Se les solicitó que valoraran cuestiones de índole general sobre gamificación (preguntas de la 1 a la 5 y la 10 y 11) y otras más específicas (preguntas de la 6 a la 9). 
1. Los test en línea facilitan aprender/repasar los contenidos y fijarlos en la memoria a través de una experiencia lúdica.

2. Fomenta la participación y la clase resulta más entretenida.

3. Es interesante como sistema de evaluación continua al facilitar la comprensión de cara a la preparación del examen final.

4. Implica agilidad y concentración por parte de los estudiantes, lo que motiva que se preste atención durante la clase.

5. El premiar con puntos, que suman en la nota final, anima a esforzarse para superar a los compañeros y de paso aprender.

6. Mi valoración sobre la herramienta de test en línea KAHOOT! es:

7. Mi valoración sobre la herramienta de test en línea SOCRATIVE es:

8. Mi valoración sobre la herramienta de test en línea QUIZIZZ es:

9. Mi valoración sobre la herramienta de test en línea GOOGLEFORMS es:

10. Valora si te hubiera gustado que se hicieran test por cada tema desarrollado.

11. Nivel de satisfacción en general con la metodología de gamificación.

En la figura 4 se muestran los resultados. Los colores asignados a los niveles de percepción de la escala son: 1-Muy malo (azul claro), 2-Malo (morado), 3-Normal (verde), 4-Bueno (burdeos) y 5-Muy bueno (azul).

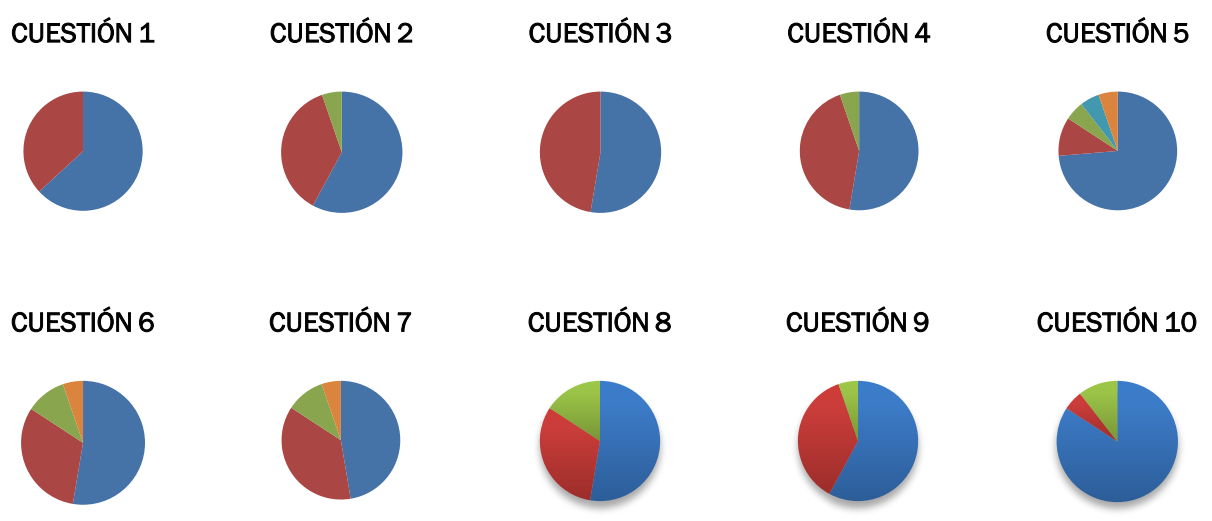

CUESTIÓN 11

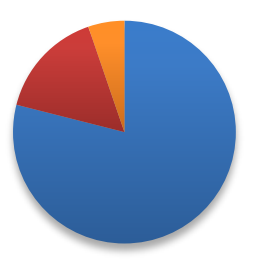

Muy bueno $\square$ Bueno $\square$ Normal $\square$ Malo $\square$ Muy malo $\square$ No contesta

Fig. 4 Encuesta de satisfacción 
Aunque algún alumno no respondió a alguna pregunta, la mayor parte puntuaron todas con valores 4 y 5 . Solamente un alumno respondió con una valoración de 1 a la pregunta 5 .

En relación a las herramientas analizadas ninguna obtuvo valoración negativa. Los alumnos las puntuaron de forma muy similar, con lo que no es sencillo en base a las respuestas decidir la más conveniente. Si nos ceñimos a los resultados, cualquiera es adecuada para la evaluación participativa o la autoevaluación.

Algunas características de las herramientas se reflejan en la siguiente tabla:

Tabla 1. Caractetísticas principales de algunas herramientas de gamificación

\begin{tabular}{|l|l|l|l|l|}
\hline & KAHOOT! & SOCRATIVE & QUIZIZZ & GOOGLEFORMS \\
\hline FORMATO & Opción múltiple & $\begin{array}{l}\text { Opción múltiple }+ \\
\text { Respuesta corta }\end{array}$ & Opción múltiple & $\begin{array}{l}\text { Opción múltiple + Respuesta } \\
\text { corta+Respuesta larga }\end{array}$ \\
\hline TIEMPO & $\leq 30 \mathrm{sg}$ & Controlado & 30 seg o ilimitado & Ilimitado \\
\hline RITMOS & Por profesor y alumno & Por el profesor & Por alumno & Por alumno \\
\hline ORDEN & Fijo o aleatorio & Fijo o aleatorio & Fijo o aleatorio & Fijo o aleatorio \\
\hline REPOSITORIOS & Sí & Sí & Sí & No \\
\hline $\begin{array}{l}\text { MÓVIL O/Y } \\
\text { ORDENADOR }\end{array}$ & Sí & Sí & Sí & Sí \\
\hline CONECTIVIDAD & Sí & Sí & Sí & Video/imágenes \\
\hline MULTIMEDIA & Video/imágenes & Imágenes & & \\
\hline
\end{tabular}

Fuente: Propia (2019)

Entre las ventajas que ofrecen destaca que son software gratuito y fácilmente accesibles. La implementación en el aula resulta rápida y el uso de los recursos educativos es sencillo. Al profesor le resulta fácil efectuar un seguimiento de los estudiantes contrastando sus avances. Incrementa el grado de motivación e interactividad al fomentar la participación de los estudiantes. El manejo de estas aplicaciones es simple, intuitivo y amigable, y se adecúan por igual a cualquier nivel académico o área de estudio. La experiencia docente es eficiente y transferible a otras materias. Se puede incluso hacer uso de cuestionarios compartidos en la base de datos por otros usuarios. También conviene aprovechar los recursos multimedia como imágenes si resulta útil.

Con relación al número de preguntas dependerá del tema, aunque no es recomendable superar las 25 para evitar que los alumnos pierdan interés. El profesor tiene la opción de descargar las respuestas del cuestionario en una hoja excel para la evaluación.

A modo de resumen destacar que:

- Kahoot! sólo permite preguntas de opción múltiple y el número de caracteres tanto de la pregunta como de las respuestas está limitado. Eso obliga a replantear las 
preguntas largas, que en ocasiones también puede ser una ventaja si el tiempo es reducido. Al final de cada pregunta se muestra la correcta.

- Socrative además de preguntas de opción múltiple, admite cuestionarios donde es posible seleccionar más de 1 respuesta y escribir texto. El profesor marca el ritmo, proyectando las cuestiones y repasando aquellas que presenten dudas. También las exit cards facilitan que los alumnos den feedback al profesor, que muestren su nivel de comprensión y presenten sugerencias.

- Estas aplicaciones requiren de un dispositivo por parte del alumno y conectividad. Esta carencia se puede suplir con otra aplicación, Plickers, donde el docente es el único que requiere de móvil para proyectar las preguntas desde del ordenador del aula (Plickers, 2016).

\section{Conclusiones}

El juego es un proceso social que mejora la motivación y fomenta el aprendizaje en diferentes niveles educativos y edades. En todas las disciplinas académicas se pide impulsar la creatividad en el aula y el uso de tecnología efectiva en la enseñanza. Para los educadores puede resultar una tarea ingrata, y desalentadora, encontrar plataformas de aprendizaje competitivas o basadas en juegos adaptadas a sus necesidades. Por encima de todo los educadores deben considerar elementos que motiven, y elegir la herramienta en base a reforzar los conceptos. Hay que ser cuidadoso para añadir las mejores preguntas.

En el presente estudio analizamos algunas de las herramientas de gamificación más conocidas. Con ello pretendemos dar a conocer sus características, pros y contras, de cara a facilitar la elección. Tras el análisis se han seleccionado cuatro para su aplicación al proceso de aprendizaje participativo en el aula y autoevaluación individual. Al ser valoradas por los discentes de forma similar, no destacando ninguna por encima de las otras, queda a criterio del alumno y profesor usar la más adecuada a sus intereses.

Podemos utilizar estas herramientas, antes del inicio de la materia, para sondear el nivel previo de conocimiento de los alumnos y ajustar en consecuencia el temario, la profundidad de los contenidos y rapidez de impartición. Los incentivos como otorgar puntos extra, al que mejor puntúe en los test, motiva y reduce el absentismo.

Los datos de la encuesta entre los alumnos que participaron en el estudio (un total de 19), encontraron estas herramientas motivadoras y facilitadoras del aprendizaje. Los miles de estudios contrastados demuestran que mejora la comprensión de contenidos, siendo las recompensas elementos cruciales para el éxito. Los resultados de gamificar muestran mejoras en la adquisición de conocimientos, en la participación, y una elevada aceptación y satisfacción entre el alumnado. Al estar más motivados prestan más atención y eso redunda positivamente en sus calificaciones, respecto a los resultados de otros cursos donde se aplicaba la metodología expositiva.

En próximos cursos se probarán nuevas aplicaciones tecnológicas tales como Pear Deck, WordDrop, Crosswords y Piktochart. 


\section{Agradecimientos}

Este trabajo no hubiera sido posible sin el apoyo proporcionado por el Vicerrectorado de Docencia de la Universidad de Valladolid (Proyecto de Innovación Docente No 145).

\section{Referencias}

BARNES, R. (2017). "Kahoot! in the Classroom: Student Engagement Technique" en Nurse Educator, vol. 42, issue 6, p. 280-280.

CHAIYO, Y.; NOKHAM, R. (2017). "The Effect of Kahoot, Quizizz and Google forms on the Student's Perception in the Classrooms Response System". En: 2017 International Conference on Digital Arts, Media and Technology (ICDAMT) - Digital Economy for Sustainable Growth. pp. 178182. Chiang Mai.

FUß, C.; STEUER, T.; NOLL, K.; MIEDE, A. (2014). "Teaching the Achiever, Explorer, Socializer, and Killer - Gamification in University Education”. En: Göbel, S., Wiemeyer, J. (Eds.), Games for Training, Education, Health and Sports, GameDays 2014. Lecture Notes in Computer Science, vol. 8395, pp. 92-99. Springer, Cham. Disponible en https://link.springer.com/chapter/10.1007/978-3-31905972-3 11\#citeas [Consulta: 10 de marzo de 2019]

GARCIA-GARCIA, D.; CARBONELL-VERDU, A.; MONTAÑES, N.; QUILES, L.; FOMBUENA, V. (2017). "Incorporación de la aplicación Kahoot! para la evaluación de las prácticas de la asignatura de "Ciencia de Materiales". Fernández Prada, M. Á. y Botti Navarro, V. J. (coord.) En: IN-RED 2017. Congreso nacional de innovación educativa y de docencia en red. pp. 1209-1217. Valencia: Editorial Universitat Politècnica de València. Disponible en http://hdl.handle.net/10251/86908 [Consulta: 20 de marzo de 2019]

GOOGLEFORMS <https://www.google.com/forms/about/> [Consulta: 3 de marzo de 2019]

GUARDIA, J. J.; DEL OLMO, J. L.; ROA, I.; BERLANGA, V. (2019) "Innovation in the teachinglearning process: the case of Kahoot!" en On the Horizon, vol. 27, issue 1, p. 35-45.

IWAMOTO, D. H.; HARGIS, J.; TAITANO, E. J.; VUONG, K. (2017) "Analyzing the efficacy of the testing effect using Kahoot (TM) on student performance" en Turkish Online Journal of Distance Education, vol. 18, issue 2, p. 80-93.

IZQUIERDO-ALVAREZ, V.; LAHUERTA-OTERO, E.; CORDERO-GUTIERREZ，R. (2018) "Kahoot, win the learning race". García Peñalvo, F. J. (coord.) En: TEEM'18 Proceedings of the Sixth International Conference on Technological Ecosystems for Enhancing Multiculturality. pp. 737-741. Salamanca. Editorial ACM New York, NY, USA.

JURGElAitis, M.; CEPONIENE, L.; CEPONIS, J.; DRUNGILAS, V. (2019) "Implementing gamification in a university-level UML modeling course: A case study" en Computer Applications in Engineering Education, vol. 27, issue 2, p. 332-343.

KAHOOT!. <https://kahoot.com/> [Consulta: 22 de marzo de 2019]

LLERENA, E. G.; RODRIGUEZ, C. P. (2017) "Kahoot! A Digital Tool for Learning Vocabulary in a language classroom" en Revista Publicando, vol. 4, issue 12, p. 441-449.

PLICKERS (2016) https://natalialzam.wordpress.com/2016/04/28/plickers-aplicacion-preguntasopcion-multiple-en-el-aula/ [Consulta: 11 de marzo de 2019] 
QUIZIZZ < https://quizizz.com/> [Consulta: 13 de marzo de 2019]

SAINZ-DE-ABAJO, B.; DE LA TORRE-DÍEZ, I.; LÓPEZ-CORONADO, M.; COUTO, C. A.; DE CASTRO-LOZANO, C. (2018). "Gamificación en la evaluación dinámica de contenidos de la materia básica del Grado en Criminología". Vega Carrero, V. y Vendrell Vidal, E. (coord.) En: $I N$ RED 2018. Congreso nacional de innovación educativa y docencia en red. pp. 804-813. Valencia: Editorial Universitat Politècnica de València. Disponible en http://dx.doi.org/10.4995/INRED2018.2018.8724 [Consulta: 15 de marzo de 2019]

SOCRATIVE $<$ https://socrative.com/> [Consulta: 23 de marzo de 2019]

SONG, Y. (2014). "Bring Your Own Device (BYOD)" for seamless science inquiry in a primary school" en Computers \& Education, vol. 74, p. 50-60.

WANG, A. I.; LIEBEROTH, A. (2016). "The Effect of Points and Audio on Concentration, Engagement, Enjoyment, Learning, Motivation, and Classroom Dynamics Using Kahoot!” En: Proceedings of the 10th European Conference on Games-Based Learning. pp. 738-746. Paisley.

WEE, S. C.; CHOONG, W. W. (2019) "Gamification: Predicting the effectiveness of variety game design elements to intrinsically motivate users' energy conservation behaviour" en Journal of Environmental Management, vol. 233, p. 97-106. 